was $92.3 \%$, with a negative predictive value of $98.8 \%$. Conclusion: A SLN biopsy in endometrial cancer has a high detection rate and high accuracy. However, surgical expertise and a learning curve are required.

Oral (OM6)

Uterine Sarcoma

https://doi.org/10.3802/jgo.2021.32.S1.0M6

\section{Impact of power morcellation and tissue extraction technique in women with unexpected uterine cancer after uterine- conserving surgery}

Hyun-Woong Cho, Jae Kwan Lee, Jin Hwa Hong*

Korea University Guro Hospital, Seoul, Korea (jhhong93@korea.ac.kr)

Objective: Although there is a concern about laparoscopic uterus-conserving surgery (UCS) including power morcellation which may adversely affect outcome in patients with unexpected uterine cancer, the impact of power morcellation and tissue extraction technique on prognosis is still indeterminate based on existing evidence. The aim of this study is to evaluate the impact of type of surgery on prognosis with interest in the change of morcellation technique.

Methods: Using Korea National Health Insurance (KNHI) database, we identified 1,788 women with unexpected uterine cancer who underwent a hysterectomy or UCS for presumed benign disease from January 2003 to December 2018. The comparison of mortality between hysterectomy and UCS was assessed according to laparoscopic and open surgery. Cox proportional hazards models were used to estimate the adjusted hazard ratios (aHRs) and $95 \%$ confidence intervals (CIs) for the mortality of patients. Results: Laparoscopic UCS was associated with a higher risk for mortality than laparoscopic hysterectomy ( $\mathrm{aHR}=3.03$; 95\% $\mathrm{CI}=1.25-7.34)$, whereas there was no significant association between open hysterectomy and UCS. The survival of laparoscopic UCS after Food and Drug Administration warning in 2014 was higher than that of laparoscopic UCS before 2014 (4-year survival rate: $100 \%$ [7/7] vs. $77.4 \%$ [24/31]). There was no significant difference of survival rate between open UCS before and after 2014.

Conclusion: Uncontained power morcellation could be associated to increased risk of mortality in women with unexpected uterine cancer. Preoperative evaluation of patents is required to reduce the effect of unexpected uterine cancer. For women who incidental uterine cancer is suspected, careful surgical procedures that can prevent tumor dissemination may improve the prognosis.
Oral (OM7)

Endometrial Hyperplasia, Endometrial Intra-epithelial Neoplasia, and Endometrial Cancer

https://doi.org/10.3802/jgo.2021.32.S1.0M7

\section{Clinicopathological factors and IHC markers predicting survival outcomes of uterine endometrial carcinoma: experience of a gynaecologic oncology unit from India}

\author{
Megha Nandwani, ${ }^{1, *}$ Hemlata Garg, ${ }^{2}$ Satinder Kaur ${ }^{3}$ \\ 'Narayana Superspeciality Hospital, Gurugram, India \\ (megha.nandwani@gmail.com) \\ ${ }^{2}$ Narayana Superspeciality Hospital, Dharamshila, India \\ ${ }^{3}$ Narayana Superspeciality Hospital, New Delhi, India
}

Objective: To study the clinicopathological factors and immunohistochemistry (IHC) markers in predicting survival outcome of women with endometrial carcinoma undergoing primary surgery. Methods: All cases of carcinoma endometrium diagnosed and treated at our hospital from 1st July 2013 to 30th June 2020 were studied and retrospectively reviewed for demographic profile and survival analysis. The factors influencing survival were critically evaluated. All histopathology slides were made to undergo IHC staining with p53 marker and microsatellite instability (MSI) markers (MLH1, MSH2, MSH6) and its correlation with survival was analyzed. Results: A total of 84 cases of carcinoma endometrium were evaluated. More than $50 \%$ patients belonged to age group of 60 years and above and were postmenopausal. The factors influencing survival were evaluated using multivariate analysis. These factors were age, stage, myometrial invasion and lymph node metastasis. The overall survival of our study population was $91.5 \%$. IHC markers like p53 and MLH1, MSH2, MSH6 were useful in predicting survival outcomes and prognosis in our study population.

Conclusion: Endometrial cancer have good survival outcomes when diagnosed and treated at an earlier stage. Factors influencing survival include age, myometrial invasion and lymph node metastasis. IHC markers; p53 and MSI markers like MLH1, MSH2, MSH6 are helpful in not only predicting survival outcome but also in guiding adjuvant treatment in these patients.

Oral (OM8)

Endometrial Hyperplasia, Endometrial Intra-epithelial Neoplasia, and Endometrial Cancer

https://doi.org/10.3802/jgo.2021.32.S1.0M8

\section{Retreatment with progestin for recurrence after achieving complete response with fertility sparing hormonal treatment in patients with early endometrial cancer}


A Jin Lee, ${ }^{1}$ Nae Ri Kim, ${ }^{1}$ Eun Jung Yang, ${ }^{1}$ Kyeong A So, ${ }^{1}$ Sun Joo Lee, ${ }^{1}$ Ji Young Lee, ${ }^{1}$ Tae Jin Kim, ${ }^{1}$ Soon-Beom Kang, ${ }^{2}$ Seung-Hyuk Shim ${ }^{1, *}$

'Konkuk University School of Medicine, Seoul, Korea

(nastassja@hanmail.net)

${ }^{2}$ Hosan Women Hospital, Seoul, Korea

Objective: To assess the outcomes of retreatment using progestin in patients with recurrent endometrial cancer (EC) after achieving complete response (CR) with fertility sparing hormonal treatment. Methods: We reviewed retrospectively patients who had recurrence after achieving CR by fertility sparing treatment using progestin with presumed stage IA, grade 1, endometrioid EC. All recurrent patients were given medroxyprogesterone acetate or megestrol acetate with concurrent LNG-IUD. The primary end point was the response rate. The secondary end points were pregnancy outcome, pathologic disease progression, and 2 nd recurrence rate.

Results: Of 55 recurrent patients, 50 received progestin retreatment. With median retreatment duration of 8 (3-56) months, the CR rate of the retreatment group was $78 \%$ (39/50); $76.2 \%$ in EC group and $87.5 \%$ in endometrial intraepithelial neoplasia group. Of 11 patients not achieving CR to progestin retreatment, 3 patients underwent surgical treatment and none had extrauterine spread of disease. Of 20 patients who attempted to conceive after achieving CR, 8 patients became pregnant. Of the 39 patients achieving CR after 1st recurrence, 20 (51.2\%) patients had 2nd recurrence with a median follow-up duration of 15 (9-146) months. Of those, 16 patients received second-line progestin retreatment and 11 (68\%) of these patients achieved CR. All patients who underwent retreatment are alive without evidence of disease.

Conclusion: First-line and second-line progestin retreatment in patients with recurrent EC are as effective and safe as initial fertility sparing treatment. Therefore, it may provide an opportunity for young patients who still want to preserve fertility after recurrence.

Oral (OM9)

Endometrial Hyperplasia, Endometrial Intra-epithelial Neoplasia, and Endometrial Cancer

https://doi.org/10.3802/jgo.2021.32.S1.0M9

\section{Indirect Excess Dose Volume Ratio (iRex): a potential toxicity controlling parameter for image-guided adaptive brachytherapy in inoperable endometrial cancer}

Tissana Prasartseree, " Pittaya Dankulchai

Division of Radiation Oncology, Department of Radiology, Faculty of Medicine, Siriraj Hospital, Mahidol University, Bangkok, Thailand (tissana.p@gmail.com)
Objective: Radiation toxicities still occurred despite achievable dose constraints in image-guided adaptive brachytherapy (IGABT) of inoperable endometrial carcinoma. Indirect Excess Dose Volume Ratio (iRex; doi: 10.5114/jcb.2020.100377) was explored for its potential toxicity restriction.

Methods: All inoperable endometrial cancer cases receiving IGABT with/without external beam radiation therapy (EBRT) in 2013-2017 were reviewed for loco-regional control, distant metastasis-free, cancer-specific, and overall survival (OS) rate. iRex60 were genuinely generated for radiation toxicity analysis, in addition to conventional brachytherapy constraints $\left(D_{2 c c}\right)$. Descriptive, Kaplan-Meier, Mann-Whitney U test, and net reclassification index (NRI), were utilized.

Results: Thirteen inoperable endometrial cancer patients (median age 74 years old, follow-up time 43 months) underwent IGABT alone and IGABT+EBRT for 1 and 12 patients. Four-year locoregional control, distant metastasis free survival, causespecific survival were all $100 \%$, but OS was $83.9 \%$. Crude grade $\geq 3$ gastrointestinal (GI) toxicity occurred in $3(23.1 \%)$ patients, yielding accrual 4-year incidence of $15.4 \%$, whereas late-persistent toxicity was presented in only $1(7.7 \%)$ patient. No high-grade genitourinary toxicity occurred. For dosimetric data (12 patients), comparing grade 3-5 with 0-2 late GI toxicity group, mean $\mathrm{D}_{2 \mathrm{cc}}$ rectum were 62.0 (range 51.7-76.1) and 58.7 (52.2-73.3) Gy EQD ${ }_{2,3}(\mathrm{p}=0.78)$, while mean iRex60 were 2.86 $(2.02-4.03)$ and $1.94(1.13-2.96)(\mathrm{p}=0.12)$, respectively. iRex- $\mathrm{D}_{2 c c}$ scatter plot showed lower toxicity incidence underneath iRex cut-point of 2. However, absolute and additive NRI of $-8.3 \%$ $(\mathrm{p}=0.93), 33.3 \%(\mathrm{p}=0.51)$ showed no significant improvement of toxicity prediction with iRex60 incorporation.

Conclusion: iRex60 showed potential cut-point of 2 from iRex$\mathrm{D}_{2 \mathrm{cc}}$ scatter plot, lower iRex60 seems to be associated with lower toxicity incidence, yet not statistically significant. Larger sample is required for validation.

\section{Ovarian cancer}

Oral (001)

Epithelial Ovarian Cancer including Borderline Tumor https://doi.org/10.3802/jgo.2021.32.S1.001

\section{Efficacy and safety of niraparib combined with oral etoposide in platinum-resistant/ refractory ovarian cancer (PROC): a single arm, phase 2 study}

\author{
Jiaxin Yang,,$^{1, *}$ Huimei Zhou, ${ }^{1}$ Qingshui Li, ${ }^{2}$ Depu Zhang, ${ }^{2}$ \\ Dongyan Cao,' Yang Xiang' \\ 'Peking Union Medical College Hospital, Chinese Academy of
}

